\title{
Dor fantasma em pacientes submetidos à amputação: revisão integrativa
}

A dor fantasma é citada desde o século XV, sendo descrita pela primeira vez em 1866 como sensação ao membro removido e sua titulação dor fantasma redefinida entre 1871-1872. O objetivo deste estudo foi identificar na literatura a semiologia, tratamento e os cuidados de enfermagem ao paciente submetido à amputação e que apresenta dor fantasma. Trata-se de uma revisão integrativa, realizada por meio das seguintes bases secundárias: coleção Scientific Eletronic Library Online (SCIELO), Literatura Latino-Americana e do Caribe em Ciências da Saúde (LILACS) e o Google Acadêmico, a partir dos descritores: 'Dor' and 'Dor Fantasma' and 'Amputação' and 'Enfermagem'. Como critérios de inclusão adotou-se: textos completos realizados no período de 1990 a 2014 , nos idiomas português e inglês. Os artigos selecionados foram dispostos num quadro síntese segundo as variáveis: autor, revista com o ano, título do artigo e objetivo geral do estudo. Foram incluídos estudos com texto completos, publicados no período de 1990 a 2014, n os idiomas português e inglês. Os estudos foram agrupados em três categorias: pacientes amputados que tiveram dor fantasma, tratamentos utilizados nesse controle e o papel de enfermagem para com estes. Foram recuperados 40 artigos na base de dados, dos quais 30 foram excluídos. Após leitura minuciosa, foram eleitos 11 artigos que atenderam aos critérios de elegibilidade definidos no estudo. A dor fantasma é um problema recorrente entre pacientes submetidos a amputação. Embora este tema seja relevante para os profissionais da saúde, poucos estudos vem trazendo a semiologia, tratamento e cuidados de enfermagem.

Palavras-chave: Amputação; Dor; Dor Fantasma; Enfermagem.

\section{Phantom pain in patients submitted to amputation: integrative review}

\begin{abstract}
Phantom pain has been mentioned since the 15th century, being described for the first time in 1866 as sensation to the removed limb and its phantom pain titration redefined between 1871-1872. The aim of this study was to identify in the literature semiology, treatment and nursing care for patients who have undergone amputation and who present with phantom pain. This is an integrative review, carried out through the following secondary bases: Scientific Electronic Collection Online (SCIELO), Latin American and Caribbean Literature in Health Sciences (LILACS) and Google Scholar, based on the descriptors: 'Pain' and 'Phantom Pain' and 'Amputation' and 'Nursing'. The inclusion criteria adopted were: complete texts carried out from 1990 to 2014, in Portuguese and English. The selected articles were arranged in a summary table according to the variables: author, magazine with the year, title of the article and general objective of the study. Fulltext studies, published from 1990 to 2014, in Portuguese and English were included. The studies were grouped into three categories: amputee patients who had phantom pain, treatments used in this control and the nursing role for them. 40 articles were retrieved from the database, of which 30 were excluded. After thorough reading, 11 articles were selected that met the eligibility criteria defined in the study. Phantom pain is a recurring problem among patients undergoing amputation. Although this topic is relevant for health professionals, few studies have been bringing semiology, treatment and nursing care.
\end{abstract}

Keywords: Amputation; Phantom Pain; Pain; Nursing.

Topic: Enfermagem Geral

Reviewed anonymously in the process of blind peer
Received: 22/02/2020

Approved: 23/05/2020
Mariane Cristina Estevão (iD

Instituto Cleber Leite, Brasil http://lattes.cnpq.br/3629022212470280

http://orcid.org/0000-0002-7138-8001 tpsi@hotmail.com

\section{Christiano Miranda (D)}

Universidade Cruzeiro do Sul, Brasil

http://lattes.cnpq.br/4631433999709517

http://orcid.org/0000-0003-2616-8744

chmiranda1977@gmail.com

Ana Paula Gomes Soares Pereira (id

Universidade Estadual de Minas Gerais, Brasil

http://lattes.cnpq.br/3631825469916595

http://orcid.org/0000-0002-1050-6035

anapaulagsoares@yahoo.com.br

\section{Elvis das Neves de Souza (iD \\ Centro Universitário Ages, Brasil http://lattes.cnpq.br/2100716023682132 http://orcid.org/0000-0001-8652-4603 elvis.nsouza@hotmail.com}

Renan Sallazar Ferreira Pereira (it

Universidade Federal do Tocantins, Brasil

http://lattes.cnpq.br/815432637102970

http://orcid.org/0000-0001-5140-4046

renansallazar@gmail.com

\section{Cyntia Ferreira Ribeiro (iD}

Faculdades Unidas do Norte de Minas Gerais, Brasil

http://lattes.cnpq.br/2329571085145222

http://orcid.org/0000-0001-9718-3090

cyntiaribeiror@yahoo.com.br
Allan Ulisses Carvalho de Melo (it) Universidade Federal da Paraíba, Brasil http://lattes.cnpq.br/2808018167548455 http://orcid.org/0000-0002-0083-6882 allanulisses@gmail.com
Referencing this:

ESTEVÃO, M. C.; MIRANDA, C.; PEREIRA, A. P. G. S.; SOUZA, E. N.; PEREIRA, R. S. F.; RIBEIRO, C. F.; MELO, A. U. C.. Dor fantasma em pacientes submetidos à amputação: revisão integrativa. Medicus, v.2, n.2, p.1-5, 2020. DOI: http://doi.org/10.6008/CBPC2674$\underline{6484.2020 .002 .0001}$
DOI: 10.6008/CBPC2674-6484.2020.002.0001 


\section{INTRODUÇÃO}

A dor pode ser conceituada como uma experiência singular e universal de características próprias e influenciada pela cultura, situações passadas, fatores físicos, emocionais e espirituais. A dor é considerada como o $5^{\circ}$ sinal vital e pode ser classificada pelo conceito temporal ou conforme seu mecanismo de ação, ou seja, pode ser classificada em dor aguda, crônica quando relacionada ao tempo, e nociceptiva, neuropática ou mista pelo mecanismo (ESTEVES, 2010).

A dor fantasma é citada desde o século XV, sendo descrita pela primeira vez em 1866 como sensação ao membro removido e sua titulação dor fantasma redefinida entre 1871-1872. Os mecanismos que compreendem a dor fantasma não estão atrelados apenas a um impulso e/ou estimulação, mas também de uma interação íntima das qualidades sensoriais, sensibilidade espacial dolorosa e os sistemas responsáveis pelos aspectos cognitivos e afetivos do indivíduo, uma vez que sua melhora ou piora depende do tronco cerebral e sistema límbico (PROBSTNER et al., 2006; TICIANELLI et al., 2003).

Nesse contexto, a prática de enfermagem pode ser dificultada devido à escassez de material de apoio técnico-científico. Para subsidiar uma assistência integral ao paciente com dor fantasma é necessário que o enfermeiro conheça a sua semiologia, tratamentos realizados e diagnóstico de enfermagem (CHAMLIAN et al., 2014). Neste sentido, o objetivo deste estudo O objetivo deste estudo foi identificar na literatura a semiologia, tratamento e os cuidados de enfermagem ao paciente submetido à amputação e que apresenta dor fantasma.

\section{MÉTODOS E MATERIAS}

Trata-se de uma revisão integrativa da literatura, realizada nas bases de dados nacionais e internacionais, com o objetivo de sintetizar resultados obtidos em pesquisas já realizadas sobre a dor fantasma. Sendo assim, tais bases de dados apresentam publicações cientificas criteriosas e confiáveis, além de ter alcance no âmbito mundial, como estudos permitem acesso múltiplo, integridade de informações, resposta rápida e melhor gestão das informações tais como: Literatura Latino-Americana e do Caribe em Ciências da Saúde (LILACS) e Medical Literature Analysis and Retrieval Sistem on-line (Medline) e o SCIELO.

Definiu-se como critérios de inclusão: artigos publicados nos anos de 1990 a 2014; com texto disponível na íntegra e em português; abordar no resumo e/ou no título características e/ou aspectos sobre dor; dor fantasma; amputação; enfermagem. A coleta de dados ocorreu no período de Agosto de 2015 a Março de 2016, utilizando as seguintes palavras chaves, associado aos operadores booleanos: 'Dor' and 'Dor fantasma' and 'Amputação' and 'Enfermagem'.

\section{RESULTADOS E DISCUSSÃO}

Foram recuperados 40 artigos a partir das bases de dados, dos quais 29 foram excluídos. Após leitura minuciosa, foram eleitos 11 artigos que atenderam aos critérios de elegibilidade, conforme apresentado no quadro 1. 
Quadro 1: Distribuição de estudos que atenderam os critérios de elegibilidade.

\begin{tabular}{|c|c|c|c|}
\hline Autor & Revista/ ano & Título & Objetivo \\
\hline Bottega et al. & $\begin{array}{l}\text { Texto \& Contexto } \\
\text { Enferm., } \\
\text { Florianópolis } \\
\text { Scielo, } 2010 .\end{array}$ & $\begin{array}{l}\text { A dor como quinto sinal vital: } \\
\text { utilização da escala de } \\
\text { avaliação por enfermeiros de } \\
\text { um hospital geral. }\end{array}$ & $\begin{array}{l}\text { Descrever as impressões dos enfermeiros sobre o } \\
\text { uso de uma escala visual analógica de avaliação da } \\
\text { dor em adultos. }\end{array}$ \\
\hline Chamlian et al. & $\begin{array}{l}\text { ACTA Fisiatr., } \\
\text { BIREME, } 2014 .\end{array}$ & $\begin{array}{l}\text { Dor relacionada à amputação e } \\
\text { funcionalidade em indivíduos } \\
\text { com amputação de membros } \\
\text { inferiores. }\end{array}$ & $\begin{array}{l}\text { Investigar a presença de dor relacionada à } \\
\text { amputação nos pacientes amputados de membros } \\
\text { inferiores em tratamento de reabilitação, avaliar } \\
\text { seus status funcionais, sem e com próteses e } \\
\text { verificar se há associação entre a presença de dor e } \\
\text { a função de marcha. }\end{array}$ \\
\hline Chamlian et al. & $\begin{array}{l}\text { ACTA Fisiatr., } \\
\text { BIREME, } 2012 .\end{array}$ & $\begin{array}{l}\text { Prevalência de dor fantasma } \\
\text { em amputados do Lar Escola } \\
\text { São Francisco. }\end{array}$ & $\begin{array}{l}\text { Avaliar a prevalência de dor fantasma na população } \\
\text { de pacientes atendidos no Centro de Reabilitação } \\
\text { Lar Escola São Francisco. }\end{array}$ \\
\hline Demidoff et al. & $\begin{array}{l}\text { Ciência } \\
\text { Cognição, } \\
\text { BVSAlud, 2007. }\end{array}$ & $\begin{array}{l}\text { Membro fantasma: o que os } \\
\text { olhos não veem, o cérebro } \\
\text { sente }\end{array}$ & $\begin{array}{l}\text { Abordar os diversos fatores que ocasionam a } \\
\text { sensação de membro fantasma, assim como seus } \\
\text { principais sintomas além de apresentar } \\
\text { experiências já realizadas em indivíduos portadores } \\
\text { deste fenômeno. }\end{array}$ \\
\hline Galhardo & $\begin{array}{l}\text { Rev. Dor, São } \\
\text { Paulo, } \\
\text { 2004. Aped, }\end{array}$ & $\begin{array}{l}\text { Mecanismos neurológicos da } \\
\text { dor fantasma. }\end{array}$ & $\begin{array}{l}\text { Analisar criticamente a construção da imagem do } \\
\text { corpo, da interioridade e do self a partir das } \\
\text { principais teses neurológicas de Ramachandran } \\
\text { sobre o fenômeno dos 'membros fantasmas'. }\end{array}$ \\
\hline Moraes et al. & $\begin{array}{l}\text { Rev. Dor, São } \\
\text { Paulo, } \quad \text { Scielo, } \\
\text { 2013. }\end{array}$ & $\begin{array}{l}\text { Bloqueio do sistema nervoso } \\
\text { simpático para tratamento de } \\
\text { dor do membro fantasma. } \\
\text { Relato de caso. }\end{array}$ & $\begin{array}{l}\text { Apresentar um caso no qual o bloqueio do sistema } \\
\text { nervoso simpático foi utilizado como adjuvante no } \\
\text { tratamento da dor do membro fantasma. }\end{array}$ \\
\hline Oliveira & $\begin{array}{l}\text { Análise } \\
\text { Psicológica, } \\
\text { Scielo, } 2000 .\end{array}$ & $\begin{array}{l}\text { Elementos psicoterapêuticos } \\
\text { na reabilitação dos sujeitos } \\
\text { com incapacidades físicas } \\
\text { adquiridas. }\end{array}$ & $\begin{array}{l}\text { Compreender os elementos psicoterapêuticos na } \\
\text { reabilitação dos sujeitos com incapacidades físicas } \\
\text { adquiridas. }\end{array}$ \\
\hline Probstner et al. & $\begin{array}{l}\text { Rev. Brasileira de } \\
\text { cancerologia, } \\
\text { INCA, } 2006 .\end{array}$ & $\begin{array}{l}\text { Incidência e prevalência de dor } \\
\text { fantasma em pacientes } \\
\text { submetidos à amputação de } \\
\text { membros: revisão de literatura. }\end{array}$ & $\begin{array}{l}\text { Estudar a prevalência de dor fantasma em pacientes } \\
\text { submetidos à amputação de membros, com ênfase } \\
\text { naqueles com doença oncológica. }\end{array}$ \\
\hline Seren et al. & $\begin{array}{l}\text { Rev. da SPAGESP, } \\
\text { BVSAlud, } 2014 .\end{array}$ & $\begin{array}{l}\text { Vivencias do luto e seus } \\
\text { estágios em pessoas } \\
\text { amputadas. }\end{array}$ & $\begin{array}{l}\text { Investigar como pessoas que sofreram amputações } \\
\text { lidam com a perda de um membro físico e como } \\
\text { vivenciam o processo de luto. }\end{array}$ \\
\hline Teixeira et al. & $\begin{array}{l}\text { Rev. Med., São } \\
\text { Paulo, GEOCITIES, } \\
\text { 1999. }\end{array}$ & $\begin{array}{l}\text { Dor fantasma e no coto de } \\
\text { amputação. }\end{array}$ & $\begin{array}{l}\text { Avaliar os fatores envolvidos na dor fantasma e no } \\
\text { coto de amputação. }\end{array}$ \\
\hline
\end{tabular}

Aos artigos foram publicados nos anos de 1999, 2000, 2004, 2006, 2007, 2012, 2013 e 2014. Observase que a quantidade de publicações sobre dor fantasma ainda são baixas, apesar da relevância do tema no cenário da saúde. Os periódicos variam entre enfermagem, fisioterapia, psicologia, área oncológica e demais setores caracterizando, assim, uma necessidade de mais conhecimento sobre o tema em várias áreas da saúde.

Em relação a avaliação da dor, Bottega et al. (2010) evidenciam o quão é desafiadora essa avaliação, quando colocada frente ao quadro clínico, estágio da doença, incapacidade do cliente em se comunicar e/ou urgência iminente, mas ainda existem controversas no manejo da dor e a analgesia adequada, uma vez que, a avaliação da dor aguda difere da avaliação da dor crônica embora haja igualdade em vários aspectos. Sendo na dor aguda ênfase nas características, sequelas e no alívio. Na dor crônica a ênfase nos aspectos psicossociais e culturais, tais como estado emocional, personalidade, situação e satisfação com trabalho, histórico familiar relacionado à expressão e manejo da dor, evidenciado essas questões na dor fantasma.

A dor fantasma pode aparecer em forma de: ardor, aperto, compressão ou como uma dor intensa e 
frequente, provando assim que a dor fantasma não é resultado de somente um evento neural mais sim a interação de vários eventos neurais (DEMIDOFF et al., 2007). Comparando esses argumentos com aqueles denotados por Moraes et al. (2013), é notório que na dor fantasma existem três mecanismos envolvidos fatores periférico, medulares e cerebrais e estes podem liberar diversos estímulos, tais como, físico (dor), psicológicos (pensamento focado na amputação e a dor) e ambientais (temperatura ou mudança climática). Galhardo (2004) enfatiza que dentre as diversas teorias e suposições para tentar explicar a dor fantasma a mais aceita é a da neuromatrix proposta por Melzack que permitiu observar-se a importância do córtex durante sua reorganização funcional e somatossensorial, por esse motivo, pode-se levar em conta o que Silas Weir Mitchell em 1872 publicou e estabeleceu, "distúrbios sensoriais dos amputados são causados pelo seccionamento dos nervos cirurgicamente e sua deficiente restauração das terminações periféricas lesadas que ficavam aglomeradas de neuromas [...]" (GALHARDO, 2004).

Para que todos esses complexos organizativos sejam compreendidos, é preciso retomar aos entendimentos propostos por Seren et al. (2014), os quais deliberam que corpo na concepção do ser humano é sinônimo de identidade e um meio de contato físico e social com o meio externo tendo impacto em suas potencialidades e limitações, havendo também modificações em suas funções e sensações relacionadas a parte amputada. Um dos fatores que contribuem ao desencadeamento da dor fantasma e da sensação do membro fantasma pode ser a passa pelo luto e não aceitação da atual situação leva ao abalado psicológico

Desta forma a amputação é de fato um trauma e a vivência da dor fantasma é muitas vezes coligada a dor antes da amputação ou pelo luto e a autoimagem que o paciente/cliente faz de si, e ainda a forma que ele encarará a vida após a alta hospitalar (OLIVEIRA, 2000).

Teixeira et al. (1999), sugere que os métodos propostos para o tratamento da dor fantasma e no coto proporciona resultados insatisfatório, embora seja obvio que o esclarecimento da situação e apoio psicológico pré e pós-operatório são importantes para o cliente/paciente amputado e ainda defende que no período pós-operatório a manutenção dos doentes em ambiente adequado, o uso precoce da prótese, o retorno rápido as atividades profissionais reduzirem a ocorrência de membro e dor fantasma.

Quanto ao tratamento da dor fantasma didaticamente organizada em três modalidades dada por Probstner et al. (2006) são, medicamentosa, de apoio e cirúrgica, os medicamentosos envolvem múltiplas gama de drogas antidepressivos, bloqueadores, anticonvulsivantes, analgésicos, opióides, anti-inflamatórios não-esteroides (AINE), os tratamentos de apoio ficam para as técnicas não invasivas como estimulação elétrica nervosa transcutânea, terapia vibratória, acupuntura, hipnose, e biofreedback, quanto a cirurgia esta pode possuir resultados negativos e por isso vem sendo pouco utilizada.

Os tratamentos são diversos e predispõe-se em terapias tanto para a parte visual quanto para a parte motora, indo de exercícios que envolvem força e movimento até aplicações elétricas estando então bem explanado que é sim parte do paciente o psicológico mais outras partes do paciente deve ser trabalhado, pois o paciente deve ser visto em sua integralidade (GOSLING, 2013).

Probstner et al. (2006), também relata que a variação de pacientes e resposta aos tratamentos e sua eficácia total ou parcial ou ainda a ausência aponta fatores como: a falta de conhecimento por parte dos 
pacientes e médicos assistentes assim como também a falta de homogeneização de critérios e instrumentos de dados que caracterizam o fenômeno e as diferentes respostas terapêuticas.

O desafio à equipe multiprofissional é o grande número de doenças associadas à amputação e que podem estar coligadas a dor fantasma, tais como diabetes mellitus, hipertensão arterial sistêmica, insuficiência renal crônica entre outras (CHAMLIAN et al., 2014). Os mesmos ainda em sua pesquisa que fatores associados a dor fantasma podem perpetuar e aumentar sua intensidade sendo estes fatores a formação de neuromas, presença de dor miofacial, lesão no membro residual (coto) e no membro préamputação. $O$ enfermeiro em sua atuação tem como objetivo fazer um elo entre o paciente e o médico. Cabe ao enfermeiro reconhecer os fatores psicológico e promover meios para que o paciente conquiste a melhora do quadro clínico, frente a amputação (CHAMLIAN et al., 2012).

\section{CONCLUSÕES}

A dor fantasma é um problema recorrente entre pacientes submetidos a amputação. Embora este tema seja relevante para os profissionais da saúde, poucos estudos vêm trazendo a semiologia, tratamento e os cuidados de enfermagem ao paciente. A escassez de estudos limita o campo de atuação e consequentemente o exercício profissional.

\section{REFERÊNCIAS}

BOTTEGA, F. H.; FONTANA, R. T.. A dor como quinto sinal vital: utilização da escala de avaliação por enfermeiros de um hospital geral. Texto Contexto Enferm., Florianópolis, v.19, n.2, p.283-90, 2010.

CHAMLIAN, T. R.; SANTOS, J. K.; FARIA, C. C.; PIRRELO, M. S.; LEAL, C. P.. Dor relacionada à amputação e funcionalidade em indivíduos com amputação de membros inferiores. Acta Fisiatr., v.21, n.3, p.113-116, 2014.

CHAMLIAN, T. R.; BONILHA, M. M. M.; MACÊDO, M. C. M.; REZENDE, F.; LEAL, C. A. P.. Prevalência de dor fantasma em amputados do Lar Escola São Francisco. Acta Fisiatr., v.19, n.3, p.167-170, 2012. DOI: http://doi.org/10.5935/01047795.20120026

ESTEVES, L. S. F.. Dor. In: Saberes e práticas: Guia para ensino e aprendizado de enfermagem. 6 ed. São Caetano do Sul: Difusa, 2010. p.131-161.

DEMIDOFF, A. O.; PACHECO, F. G.; SHOLL-FRANCO, A.. Membro fantasma: o que os olhos não veem, o cérebro sente. Ciência e Cognição, v.12, p.234-239, 2007.

GABARRA, L. M.; CREPALDI, M. A.. Aspectos psicológicos da cirurgia de amputação. Aletheia, v.30, p.59-72, 2009.

GALHARDO, V.. Mecanismos neurológicos da dor fantasma. Rev. Dor, v.12, n.1, p.12-18, 2004.

GOSLING, A. P.. Mecanismos de ação e efeito da fisioterapia no tratamento da dor. Rev. Dor, São Paulo, v.13, n.1, p.6570, 2003.
MORAES, F. B.; BARBOSA NETO J. O.; VANETTI, T. K.; MORAIS, L. C.; SOUSA, Â. M.; ASHMAWI, H. A.. Bloqueio do sistema nervoso simpático para tratamento de dor do membro fantasma. Relato de caso. Rev. Dor, São Paulo, v.14, n.1, p.155-157, 2003.

PROBSTNER, D.; THULER, L. C. S.. Incidência e prevalência de dor fantasma em pacientes submetidos à amputação de membros: revisão de literatura. Rev. Brasileira de cancerologia, v.52, n.4, p.395-400, 2006.

OLIVEIRA, R. A.. Elementos psicoterapêuticos na reabilitação dos sujeitos com incapacidades físicas adquiridas. Análise Psicológica, 2000.

SADATSUNE, E. J.; LEAL, P. C.; CLIVATTI, J.; SAKATA, R. K.. Dor crônica pós-operatória: fisiologia, fatores de risco e prevenção. Rev. Dor., v.12, n.1, p.58-63, 2011. DOI: http://doi.org/10.1590/S1806-00132011000100013

SEREN, R.; TILIO, R.. Vivencias do luto e seus estágios em pessoas amputadas. Rev. da SPAGESP, v.15, n.1, p.64-78, 2014.

SILVA, S. G.. Sofrimento e suas funções para o homem. In: SIMBIDOR, 10. Anais. São Paulo, 2011. p.91-92.

TEIXEIRA, M. J.; IMAMURA, M.; CALVIMONTES, R. C. P.. Dor fantasma e no coto de amputação. Rev. Med., São Paulo, v.78, n.2, p.192-6, 1999.

TICIANELLI, J. G.; BARAÚNA, M. A.; SILVA, A. M. C.. Efeito da estimulação elétrica nervosa transcutânea na dor fantasma. Rev. Brasileira de Fisioterapia, v.7, n.2, p.115-122, 2003

A CBPC - Companhia Brasileira de Produção Científica (CNPJ: 11.221.422/0001-03) detém os direitos materiais desta publicação. Os direitos referem-se à publicação do trabalho em qualquer parte do mundo, incluindo os direitos às renovações, expansões e disseminações da contribuição, bem como outros direitos subsidiários. Todos os trabalhos publicados eletronicamente poderão posteriormente ser publicados em coletâneas impressas sob coordenação da Cognitionis Publishing, da Companhia Brasileira de Produção Científica e seus parceiros autorizados. Os (as) autores (as) preservam os direitos autorais, mas não têm permissão para a publicação da contribuição em outro meio, impresso ou digital, em português ou em tradução. 